\title{
Injured Workers Perspectives on Recovery following Non-Life-Threatening Acute Orthopaedic Trauma: A Descriptive Study
}

\author{
Fiona J. Clay, ${ }^{1}$ Swati Shourie, ${ }^{1}$ Priscilla Robinson, ${ }^{2}$ Donna McKenzie, ${ }^{3}$ and Emily Kerr ${ }^{1}$ \\ ${ }^{1}$ Injury Outcomes Research Unit, Monash Injury Research Institute, Monash University, Building 70, Level 1, Clayton Campus, \\ Melbourne, VIC 3800, Australia \\ ${ }^{2}$ School of Public Health, Faculty of Health Sciences, La Trobe University, Bundoora, Melbourne, VIC 3086, Australia \\ ${ }^{3}$ Institute for Safety Compensation and Recovery Research, Monash University, Melbourne, VIC 3004, Australia \\ Correspondence should be addressed to Fiona J. Clay; fionajclay@gmail.com
}

Received 26 October 2013; Accepted 23 December 2013; Published 23 February 2014

Academic Editors: M.-R. Lin and F.-Z. Shaw

Copyright (C) 2014 Fiona J. Clay et al. This is an open access article distributed under the Creative Commons Attribution License, which permits unrestricted use, distribution, and reproduction in any medium, provided the original work is properly cited.

\begin{abstract}
Background. Little is known about the recovery process following non-life-threatening acute orthopaedic trauma from the viewpoint of the injured person. A better understanding could facilitate optimal rehabilitative planning. Objective. To explore patients' views on factors important to them in recovery following non-life threatening acute orthopaedic trauma. Methods. Descriptive study utilizing content analysis and chi-square analysis. To better understand recovery expectations, 168 adults who had sustained non-life threatening acute orthopaedic trauma were surveyed at 2, 12, and 26 weeks after injury and invited to respond to the following question "what are the most important things necessary for you to best recover?" Results. According to participant's responses, major themes on recovery involved a return to health and a return to health but with an ongoing plan, and for a minority (12\%) recovery involved a focus on their current status. The study found that some recovery expectations changed over time. Conclusion. The journey to recovery is complex, often prolonged, and highly individual. Responses suggest that some injured persons need more assistance for a successful recovery than others. Those who appeared "caught in the moment" of the injury may benefit from clinical and rehabilitative management focusing on long-term recovery and acceptance of the injury event.
\end{abstract}

\section{Introduction}

Acute traumatic injury is a major contributor to the global burden of injury [1]. Fortunately, with the continued evolution of advanced trauma systems, an increasing number of people are surviving traumatic injuries. Despite this, the dimensions of recovery from the viewpoint of the injured person are not well understood. Recovery is often measured in terms of return to work (RTW), pain levels, and activities of daily living using standardized patient report instruments [26]. However, it is clear that these instruments only account for certain aspects of the recovery process as research indicates they do not align well with the injured person's experience of recovery.

Positive recovery expectations predict better outcomes for a variety of health conditions including recovery from whiplash-associated disorders and lower back pain [7]. Quantitative studies have measured the association of recovery expectations with time lost from work, pain, and functional status as well as factors associated with recovery expectations $[7,8]$. However, quantitative studies have limited ability to explore individual experiences and identify the finer intricacies of recovery from the point of view of the injured worker.

Qualitative research has explored recovery from the perspective of the injured person following physical trauma, lower back pain, hand injuries, and upper limb musculoskeletal disorders [9-12]. Various themes have emerged from these studies including the "importance of the injury event" and "moving on from the trauma" as well as the role of family and work support. Other studies have described recovery in terms of symptom attenuation, improved capacity to perform, and 
quality of life. Overall though, it is clear that the actual process of recovery for an injured person is a complex issue and if patient care, prognosis, and rehabilitation are to be improved then further research to address the intricacies of recovery from the viewpoint of the injured person is needed.

Injured persons' views on recovery following non-lifethreatening acute orthopaedic injuries have rarely been addressed. However, research findings indicate that the journey to recovery and independence even among those with minor traumatic injuries can be slow and vary considerably. The few studies undertaken in this area suggest that some people recover well following acute orthopaedic injuries but others have prolonged periods of poor health experiencing persistent pain, poor mental health, and extended inability to perform duties at work. With an increasing emphasis being placed on self-management of health, exploring the participant's viewpoint of recovery will be vital to empowering the patient to participate in the management of their injury.

The current analysis forms part of a prospective cohort follow-up study that examined biopsychosocial factors associated with work disability and persistent pain following nonlife-threatening acute orthopaedic trauma. The aim of the current study is to explore injured workers' views on what would aid their recovery following non-life-threatening acute orthopaedic trauma. The study was designed to establish if there were commonalities of responses provided by study participants at each particular time point, whether these responses changed with time following the injury, and the potential impact of these responses. The study is grounded in a theoretically biopsychosocial framework approach [13], that postulates that factors related to the person's own experience and understanding of their psychosocial functioning and their environment are as important as physical factors relating to the injury in determining recovery. In this paper, we present the recovery expectations of a cohort of patients with range of non-life-threatening orthopaedic injuries over time.

\section{Method}

2.1. Study Design and Setting. Full details of the study design, setting, and participants have been published elsewhere [3, 6]; however we provide a synopsis of our methods here. The Determinants of Outcome in Orthopaedic Trauma is a multicentre prospective follow-up cohort study conducted in the state of Victoria, Australia. In 2005 the year the study commenced, the population of the state of Victoria was listed as 5.02 million.

This study utilizes data collected as part of the Determinants of Outcome in Orthopaedic Trauma study. The primary focus of the main DOOT cohort study was to establish the predictive determinants of work disability and persistent pain following non-life-threatening acute orthopaedic trauma. The focus of the current analysis is to explore patient's views on important factors that would enable recovery following non-life-threatening acute orthopaedic trauma.
2.2. Participants and Procedures. Patients presenting to one of four hospitals in Victoria as a result of sustaining a nonlife-threatening acute orthopaedic trauma were recruited to the study. The Victorian Admitted Episodes Dataset (VAED) is a dataset of acute patient hospital admissions representing 100\% coverage of hospital admissions to public hospitals in Victoria [14]. The VAED was used to select study hospitals in different geographical regions in order to achieve a representative sample of Victorian working age population admitted to its public hospitals annually as a consequence of sustaining acute unintentional trauma. The hospitals selected for the study broadly reflected a range of socioeconomic status in patients admitted to hospitals. The choice of hospitals was also based on their trauma status under the Victorian State trauma system in order to facilitate the recruitment of patients with a range of orthopaedic injuries. Four hospitals took part, one regional hospital, two metropolitan hospitals, and one level 1 major trauma hospital. The level 1 trauma hospital receives more serious injuries or concerning presentations.

For the purpose of this study, an unintentional injury was defined as what could be coded as an accident using the International Statistical Classification of Disease-Australian Modification (ICD10-AM) codes "V01-V99" [15]. Injury was further defined according to the ICD10-AM codes "S00-T98" [15]. These codes do not include psychological injury.

Injury factors were retrieved from the patient's medical record in order to allow for the coding of the injury according to the Abbreviated Injury Severity (AIS) Scale [16] and the subsequent calculation of the Injury Severity Score (ISS) [17]. Patients were classed as having a minor injury if they had an ISS of 1-8, moderate injury ISS 9-15, and a major injury ISS > 15.

The inclusion criteria were people aged 18 to 64 in paid employment during the 4 weeks prior to the injury, with sufficient English language skills to allow completion of questionnaires. Patients were excluded if they had sustained an intentional injury, were not employed, or if medical staff considered them to be medically unfit to provide informed consent. Patients with a significant traumatic brain injury associated with prolonged loss of consciousness were excluded because of the documented cognitive sequelae that are not comparable to other types of injury.

Patients were recruited following presentation to the hospital emergency department. After obtaining informed consent, demographic and occupation data was collected and a retrospective assessment of preinjury health was conducted at recruitment. In addition, patients were further surveyed by phone or in person if they were still in hospital, 2 weeks, 12 weeks, and 26 weeks following their injury. Follow-up time-points were chosen based on the different phases of disability (acute, subacute, and chronic) following injury. All participants for this study were recruited and followed up between March 2005 and October 2006.

2.3. Injury Compensation in the State of Victoria. In the Australian state of Victoria, all employers are required to have workers' compensation insurance. Employers maintain this 
insurance either through the Victorian WorkCover Authority (VWA) unless they are entitled to self-insure or obtain insurance through Comcare, the national workers' compensation scheme. The majority of Victorian workers (85\%) are insured by the VWA. A claim may be lodged once the employee has been off work for 10 days or if a medical excess threshold has been exceeded. Any driver, passenger, pedestrian, motorcyclist, or cyclist that is injured on a designated Victorian road and/or in a designated vehicle is covered by the Transport Accident Commission. The injured person must meet the initial costs of medical treatment $(\$ 564.00)$ and the first week of sick leave.

Injuries with no entitlement to state-based compensation include those sustained at home, most sporting injuries, and transport accidents that occur on a nondesignated road. Selfemployed workers are not entitled to coverage by VWA or Comcare.

2.4. Study Factors and Measures. At each post-injury timepoint, participants were asked if they had returned to work. Those who answered in the affirmative were asked when they returned and if they had had any periods of sick leave since their return to work.

At recruitment, participants were asked whether they had experienced any difficulties with pain prior to the injury. Pain intensity was measured at the first followup using the short-form McGill Pain Questionnaire [18], a validated dedicated pain measurement tool [19]. Participants were asked to rate their overall pain since the injury using a 6item adjectival scale. The scale is scored: $1=$ no pain, 2 = mild, $3=$ discomforting, $4=$ distressing, $5=$ horrible, and $6=$ excruciating. Responses were dichotomised into the following groups (high: distressing/horrible/excruciating and mild: no pain/mild/ discomforting).

Participants reporting negative emotional states of depression, anxiety, and stress were assessed using the DASS21 a generic measure comprising three self-report scales [20]. The scales were scored and categorized according to recommended cut-offs (normal, mild, moderate, severe, and extremely severe) [21]. The DASS21 was collected prospectively at two weeks followup. The reported symptoms do not infer a clinical diagnosis [21].

Recovery expectations were measured at each post-injury follow-up time-point by asking participants to rate on a numerical $0-10$ rating scale if they believed they would recover enough to return to their usual preinjury activities. The recovery expectations question was derived from work by Cole et al. [22]. High scores represented strong recovery beliefs.

Compensable status was measured by asking participants if they were receiving injury compensation medical treatment or wage from state-based compensation authorities responsible for work injury and/or transport related injury.

Comorbid health conditions were obtained from the medical records pertaining to the current injury. Age was assessed as a continuous variable. Dichotomous categories defined for some factors were educational status completed university degree versus completed less than university degree, ISS scores (ISS $<9$ versus ISS $\geq 9$ ) based on the common groupings for minor, moderate, and major trauma and comorbid health conditions (none versus one or more).

Participants were asked to indicate their occupation. In some cases the response provided was not their occupation but the industry in which they were employed.

2.5. Questionnaire Data Collection and Synthesis. At each postinjury time-point, participants were invited to respond to the question: "What are the most important things necessary for you to best recover?" This was an open ended question. Participants were invited to voice the primary concerns and no coaching of responses or suggested answers were provided. A qualitative approach was taken to the analysis of these data. Responses were transcribed verbatim.

The coding process involved two stages. The open text responses were first analysed for common themes using qualitative content analysis themes by FC, EK, and SS separately $[23,24]$. As participant responses can illustrate themes, each response was at first grouped into broad emerging themes based on the common words used in their actual response. For example, the category time and rest included responses like person A who indicated "I need time to get over my injury." The category "following instructions" broadly classified responses such as those from person B who stated "doing what I am told by the physio." After this initial step, FC and SS met several times to discuss the emerging themes. Following discussions, the common themes seem to fall under eleven preliminary themes, which were then reorganised into themes that reflected a core thought process of the participant. The themes allow the researchers to bring together the different views and perspectives about recovery expectations in a structured manner and assist in exploring the journey from injury to recovery $[23,24]$.

Recovery has been described as a journey where the "event of the injury" has been used as a demarcating line categorising a patient's life into "before the trauma" and "after the trauma" [10]. In addition, taking the "event of the injury" as a starting point of recovery, the same study categorised the journey to recovery as "event" where the "event becomes a defining moment"; "fall-out" where the individuals become increasingly aware of the effect of injury, and "moving on" which involves overcoming dependence. The "event," "fall-out," and "moving-on" appear to describe the recovery process as a continuum. In our content analysis, we wanted to assess "where the attention or focus" of the participant was at each time point and how this changes over time. So in the current analysis, we examined the "focus" of the participants' responses.

2.6. Quantitative Data Analysis. Frequencies were used to report the broad themes. Chi square $\chi^{2}$ analysis $(3 \times 2)$ was used to determine significant differences in the frequency of common responses. The SPSS 19.0 program (SPSS Release 19.0, Chicago, USA) was used for all data analysis. Descriptive data are presented as means (standard deviations) or number of subjects (percentages). The baseline characteristics of patients with complete data at six months were compared 
TABLE 1: Characteristics of the study participants. Recovery expectations measured according to the question: "Do you believe you will recover enough to return to your pre-injury activities?" measured on a 0-10 Likert scale. High scores represent stronger expectations.

\begin{tabular}{|c|c|c|}
\hline \multirow{2}{*}{ Age last birthday } & \multicolumn{2}{|c|}{37.7 years $(18-62)($ mean/range $)$} \\
\hline & $N$ & $(\%)$ \\
\hline \multicolumn{3}{|l|}{ Gender } \\
\hline Male & 126 & $(75.0)$ \\
\hline Female & 42 & $(25.0)$ \\
\hline \multicolumn{3}{|l|}{ Injury severity score } \\
\hline Minor: 1-8 & 88 & $(52.4)$ \\
\hline Moderate and major: $>9$ & 80 & $(47.6)$ \\
\hline \multicolumn{3}{|l|}{ Education } \\
\hline Less than university & 136 & $(81.0)$ \\
\hline University & 32 & $(19.0)$ \\
\hline \multicolumn{3}{|c|}{ Receipt of compensation for injury* } \\
\hline No & 91 & $(55.8)$ \\
\hline Yes & 72 & $(44.2)$ \\
\hline \multicolumn{3}{|c|}{ Returned to work after 26 weeks after injury ${ }^{*}$} \\
\hline Yes & 104 & $(68.4)$ \\
\hline No & 48 & $(31.6)$ \\
\hline \multicolumn{3}{|c|}{ Pain intensity at 2 weeks after injury } \\
\hline Low pain intensity & 104 & $(61.9)$ \\
\hline High pain intensity & 64 & $(38.1)$ \\
\hline \multicolumn{3}{|c|}{ Presence of pain at 26 weeks after injury } \\
\hline Yes & 81 & $(54.0)$ \\
\hline \multirow[t]{2}{*}{ No } & 69 & $(46.0)$ \\
\hline & Mean & $\mathrm{SD}$ \\
\hline \multicolumn{3}{|l|}{ Recovery expectations $^{\#}$} \\
\hline Week $2(n=161)$ & 8.60 & 1.90 \\
\hline Week $12(n=148)$ & 8.49 & 1.92 \\
\hline Week $26(n=108)$ & 7.77 & 2.07 \\
\hline
\end{tabular}

${ }^{*}$ Missing responses. ${ }^{\#}$ Significant difference $P<0.05$ : week 2 versus week 26 and week 12 versus week 26.

with participants with incomplete followup using chi-square tests or $t$-tests. A probability $(P)$ value of $<0.05$ was considered significant.

2.7. Ethics. The research was conducted in accordance with the Declaration of Helsinki [25]. The study and all interviews were conducted with the understanding and consent of all study participants. Ethics approval was obtained from the Standing Committee on Ethics in Research Involving Humans of Monash University and the corresponding Ethics Committees at all participating hospitals.

\section{Results}

3.1. Demographics and Injury Characteristics. Baseline surveys were completed by 168 Victorian workers who had sustained nonlife-threatening acute orthopaedic trauma leading to hospitalization and were recruited to the study. Over the course of the study, 18 persons were lost to followup (89\% followup). A comparison of those lost to followup with those that remained in the study revealed no significant differences with respect to age, gender, education, compensable status, injury severity, and hospital of admission (data not shown). In addition, there was no difference in terms of hospital location with regards to those who declined to take part in the study and those who took part. The median time to followup was 17 days (2-week followup), 82 days (12-week followup), and 181 days (six-month followup).

3.2. Participants. All participants were in salaried employment at the time of their injury. The mean age of the sample was 37.7 years and the cohort consisted primarily of men $(75 \%)$. Descriptive characteristics of the study cohort are presented in Table 1. One-third of the cohort presented with one or more comorbid conditions at study entry and 91 participants (56\%) sustained an injury that provided entitlement to injury compensation. Using the ISS to classify injuries, 88 patients sustained minor injuries (ISS 1-8), 69 moderate injuries (ISS 9-15), and 11 major injuries (ISS > 15).

3.3. Outcome Measures. Return to work and pain outcomes for participants were the main outcomes in this cohort study and have been described in detail elsewhere. However, to provide context to this substudy, key findings are summarised 
TABLE 2: Grouped response to question according to follow-up time-point: What are the most important things necessary for you to best recover? Chi square analysis for differences in the frequency of the most common responses according to the three postinjury time-points.

\begin{tabular}{|c|c|c|c|c|c|c|c|c|c|}
\hline & \multicolumn{2}{|c|}{ Week 2} & \multicolumn{2}{|c|}{ Week 12} & \multicolumn{2}{|c|}{ Week 26} & \multirow{2}{*}{ Chi square } & \multirow{2}{*}{$\mathrm{df}$} & \multirow{2}{*}{$P$} \\
\hline & $N$ & $\%$ & $N$ & $\%$ & $N$ & $\%$ & & & \\
\hline Time and rest & 82 & 35.2 & 29 & 15.5 & 10 & 8.5 & 39.9 & 2 & 0.000 \\
\hline Positive attitude looking after myself & 19 & 8.1 & 29 & 15.5 & 23 & 19.5 & 10.2 & 2 & 0.006 \\
\hline Doing the therapy or exercises that have been prescribed & 37 & 15.8 & 39 & 20.9 & 29 & 24.5 & 4.13 & 2 & 0.127 \\
\hline Following instructions & 24 & 10.3 & 4 & 2.1 & 1 & 0.85 & 19.6 & 2 & 0.000 \\
\hline Further repair of the injury (including further treatment) & 23 & 9.8 & 25 & 13.4 & 8 & 6.7 & 3.57 & 2 & 0.168 \\
\hline Family support & 9 & 3.8 & 3 & 1.6 & 12 & 10.1 & & & \\
\hline Returning to work & 4 & 1.7 & 5 & 2.6 & 5 & 4.2 & & & \\
\hline Support from workplace including income support & 5 & 2.15 & 7 & 3.7 & 3 & 2.5 & & & \\
\hline Quality care & 4 & 1.7 & 6 & 3.2 & 3 & 2.5 & & & \\
\hline Get back into a normal routine & 9 & 3.8 & 6 & 3.2 & 0 & & & & \\
\hline I have recovered & & & 5 & 2.6 & 7 & 5.9 & & & \\
\hline Nothing as it will not change & & & 9 & 4.8 & 1 & 0.84 & & & \\
\hline Total number of responses & 233 & & 186 & & 118 & & & & \\
\hline Number and proportion of participants who provided responses & 161 & $(86.6 \%)$ & 148 & $(79.6 \%)$ & 108 & $(50.1 \%)$ & & & \\
\hline Mean number of responses/responding participant & 1.4 & & 1.3 & & 1.1 & & & & \\
\hline
\end{tabular}

Responses were separated for doing the therapy and exercise and following instructions. Following instructions involved no mention of therapy or exercises. The chi square analysis $(3 \times 2)$ compared each response for the three time-points. Numerator is the response under consideration e.g., time and rest, denominator all other responses, df: degrees of freedom, $P$ : probability. The bold numbers refer to the number of responses and proportions for the responses provided at 12 weeks post injury.

here. Follow-up data on return to work was available for 152 participants as 18 participants were lost to followup. Sixtyeight percent of participants $(n=104)$ returned to work within 6 months [6]. The prevalence of pain was common with nearly a half (54\%) of participants reporting the presence of persistent pain at 6 months, and over two-thirds (87\%) reporting that their pain interfered with their normal work activities [3].

At the first followup, moderate, severe, or extremely severe symptoms of depression, anxiety, and stress were reported by 32 participants (depression 19.8\%), 40 participants (anxiety $24.8 \%$ ), and 26 participants (stress $16.1 \%$ ). The levels of symptoms reported by participants as measured by the DASS21 at the first follow-up time-point are outlined in Table 5.

3.4. Recovery Expectations. Participant views on recovery, including their expectations, were measured at each followup time-point using a Likert scale from 0 to 10 on "whether they believed they would recover?" (Table 1). The mean score at each time-point indicated a statistically significant reduction in recovery expectations between two weeks and six months after injury $(P<0.05)$.

3.5. Question on Recovery-Quantitative Analysis. The open ended question revealed personal circumstances, including an insight into the feelings of patients and their views on recovery. Responses are summarised according to the timepoint at which they were provided: 2,12 , and 26 weeks postinjury (Table 2).

At week 2 after injury, the most common response to the question "important things that will help you best recover" was "time and rest" (35.2\%) followed by "doing the therapy" and exercises prescribed to them as aiding their recovery (15.5\%). Time and rest as a response was less important 12 weeks after injury. At that time-point, "doing the therapy and exercises" was the most common response $(23.4 \%)$ followed by "maintaining a positive outlook." At 26 weeks after injury these responses were again the most common with "doing therapy and exercise" scoring $28.5 \%$ of the responses and "having a positive attitude" $22.5 \%$.

Chi square analysis was used to determine if there was a statistically significant change for the most common responses over the three follow-up time-points. In this analysis, the numerator comprised the particular response, for example, "time and rest" and the denominator included the total number of all other responses provided at that timepoint. The chi-square analysis indicated a statistically significant difference in the frequency of the grouped responses over the three follow-up time-points for: (1) time and rest; (2) maintaining a positive outlook; and (3) doing what health providers told them to do. There was a nonsignificant difference in the frequency of responses for the grouped responses: (1) therapy and exercises and (2) further repair of the injury.

3.6. Open Ended Question Exploring Participants' Views on Recovery. In order to explore patients' views about what would aid their recovery following non-life-threatening acute orthopaedic trauma, content analysis was carried out on the brief open text responses provided by participants to the question "what are the most important things that will help you best recover?" Responses were broadly categorized into 
TABLE 3: Characteristics of study participants provided to add context to outlined open ended responses according to return to work status at 6 months.

\begin{tabular}{|c|c|c|c|c|c|c|c|c|c|}
\hline $\begin{array}{l}\text { Participant } \\
\text { code }\end{array}$ & Age & Gender & $\begin{array}{c}\text { Compensable } \\
\text { injury }\end{array}$ & Occupation/industry & $\begin{array}{l}\text { DASS21 } \\
\text { Symptoms } \\
2 \text { weeks }\end{array}$ & $\begin{array}{l}\text { Returned to } \\
\text { work } \\
\text { by } 6 \text { months }\end{array}$ & $\begin{array}{l}\text { Pain intensity } \\
2 \text { weeks }\end{array}$ & $\begin{array}{c}\text { Presence of } \\
\text { pain } \\
\text { at } 12 \text { weeks }\end{array}$ & $\begin{array}{l}\text { Recovery } \\
\text { beliefs } \\
\text { at } 2 \text { weeks }\end{array}$ \\
\hline RTW1 & 18 & Male & no & Bus driver & No & Yes & Low & No & 4 \\
\hline RTW2 & 18 & Female & no & Horticulture & Yes & Yes & Low & No & 9 \\
\hline RTW3 & 61 & Female & No & Bakery assistant & No & Yes & Low & Yes & 10 \\
\hline RTW4 & 47 & Female & Yes & Assembler worker & No & Yes & Low & No & 10 \\
\hline RTW5 & 58 & Female & No & Psychologist & No & Yes & Low & Yes & 10 \\
\hline RTW6 & 18 & Female & Yes & Cattery & Yes & Yes & Low & No & 6 \\
\hline RTW7 & 51 & Female & Yes & Library officer & Yes & Yes & High & Yes & 10 \\
\hline RTW8 & 54 & Male & No & Courier & Yes & Yes & Low & No & 10 \\
\hline RTW9 & 62 & Male & No & $\begin{array}{l}\text { Air conditioning } \\
\text { technician }\end{array}$ & No & Yes & Low & Yes & 9 \\
\hline RTW10 & 44 & Male & No & Manufacturing & No & Yes & Low & Yes & 5 \\
\hline RTW11 & 34 & Male & No & Construction & Yes & Yes & Low & Yes & 6 \\
\hline RTW12 & 48 & Male & No & Carpenter & No & Yes & Low & Yes & 9 \\
\hline RTW13 & 35 & Male & No & Electrical inspector & No & Yes & Low & No & 10 \\
\hline RTW14 & 41 & Male & No & $\begin{array}{l}\text { Information } \\
\text { technology }\end{array}$ & No & Yes & Low & Yes & 10 \\
\hline RTW15 & 34 & Female & No & Healthcare & No & Yes & Low & Yes & 10 \\
\hline RTW16 & 31 & Male & Yes & $\begin{array}{l}\text { Information } \\
\text { technology }\end{array}$ & Yes & Yes & Low & Yes & 7 \\
\hline NO1 & 27 & Male & no & Car industry & Yes & No & Low & No & 6 \\
\hline $\mathrm{NO} 2$ & 25 & Male & Yes & Radiographer & No & No & High & Yes & 10 \\
\hline $\mathrm{NO} 3$ & 47 & Female & No & Factory worker & No & No & Low & No & 5 \\
\hline $\mathrm{NO} 4$ & 30 & Male & Yes & Labourer & No & No & Low & Yes & 10 \\
\hline NO5 & 56 & Male & Yes & Construction & No & No & Low & No & 9 \\
\hline NO6 & 58 & Female & Yes & Nurse & Yes & No & Low & Yes & 8 \\
\hline NO7 & 49 & Male & Yes & Education & No & No & high & Yes & 10 \\
\hline NO8 & 26 & Male & Yes & Groundsman & Yes & No & High & No & 10 \\
\hline NO9 & 62 & Male & Yes & Construction & Yes & No & High & Yes & - \\
\hline NO10 & 39 & Male & Yes & Plasterer & No & No & High & Yes & 9 \\
\hline NO11 & 51 & Male & Yes & Truck driver & Yes & No & Low & Yes & 7 \\
\hline
\end{tabular}

* Participant reported symptoms of anxiety, depression, and/or stress (one or more).

three primary themes and the expression of these themes based on the participant's main focus.

3.7. Open Ended Responses on Recovery: Descriptors. While open ended responses were received from most participants, Table 3 provides the range of descriptors for those participants whose open ended response is reported in this paper. The descriptors are provided to add context and depth to responses. The choice of descriptors was based on the data collected, the biopsychosocial focus of the overall study, and the literature on factors associated with recovery.

Three key themes emerged, the first recovery focused on a return to health with an ongoing plan, the second Ideal recovery involves a focus on return to health, and the third recovery involves a focus on current status. Brief illustrative responses of the different ways of expressing the same theme are provided in Table 4 . Around half of the participants seemed to focus on their immediate situation, namely, a "return to health." A small number of participants (12\%) were focused on their current status with the remainder being those participants who had an ongoing plan for after their return to health. Individual participant comments are included in both the tables and the text and include reference to the participant code. For example, (RTW2) refers to the second participant who returned to work and (NO5) refers to the 5th participant who did not return to work as listed in Table 4.

\subsection{Recovery Themes}

3.8.1. Recovery Focused on a Return to Health with an Ongoing Plan. The first theme included those participants who 
TABLE 4: Recovery themes, subthemes, and illustrative quotes.

\begin{tabular}{ll}
\hline Themes & Subthemes \\
\hline $\begin{array}{l}\text { Focused on a return to physical } \\
\text { functioning }\end{array}$ \\
\\
$\begin{array}{l}\text { Focused on complete or partial } \\
\text { symptom resolution }\end{array}$
\end{tabular}

Recovery involves a focus on return to health
Focused on following instructions and/or advice of their health providers

Focused on healthcare, providers, and resources

Focused on others: unsure of their role

Focused on their own mental health

Focused on quality of life

Focused on recovery with an emphasis on financial or employer support

Illustrative quotes

"Turn my head more as I have a restricted range of movement" (RTW2)

"First rest, then learning how to walk on two legs again" (NO3)

"Leg healing, once that is right, I am happy" (NO8)

"Pins to come out of (leg) and for it (the bone) to knit" (RTW4)

"Hand therapy for my little finger on right hand so that I can recover movement and feeling" (NO6)

"That I follow the therapist's instructions as closely as possible" (NO12)

"Listening to advice of medical/physio people and adhering to their advice" (RTW1)

"Rest and keeping my leg elevated, don't overdo it" (RTW3)

"Better sling for my arm, one that doesn't cut the circulation off at my neck" (RTW16)

"Get strength back in my leg, different doctors all the time doesn't help me recover" (RTW14)

"Up to the doctors and physios- I don't want to start doing things without seeing the doctor" (NO11)

"Keep going back to Dr for tests and x-rays" (RTW11)

"Good healthy outlook, I need to believe that I am going to recover" (RTW9)

"Keep my mind active, feel as if I am contributing" (RTW7)

"Keep up the social activities with family and friends" (RTW15)

"Family support, enjoy life and my hobbies" (NO2)

"Having my job held open for me, not working till I am well enough " (RTW12)

"Not having the financial burden of not working" (RTW13)

"Confidence of my employer to look after me" (RTW8)

"Flexibility in working hours, understanding from my employer re mobility restrictions" (RTW5)

"Consideration at work for my temporary limitations, I will need assistance with parking” (RTW5)

"Different tasks at work, no heavy lifting" (RTW6)

"Strengthen my heel so that I get full movement back in my foot and can return to work" (RTW10)

functioning without expressed

"Being able to work again normally, get back to where I was" (NO5)

"So your injury never occurred, to turn back time" (NO9)

"Nothing will help, doctors say I won't improve" (NO7)

"Being told the truth by my doctors to allow me to heal myself" (NO4)

"My thumb needs to be perfect for me to do my job properly and I can't see that happening" (NO1) articulated a plan for return to health and had given some thought towards an ongoing plan (i.e., plans after recovery). "Consideration at work for my temporary limitations, I will need assistance with parking" (RTW5). Two different aspects emerged. In the first subtheme, the ongoing plan primarily focused around a return to physical functioning without expressed concern about their work situation or their employer. "Being able to work again normally, get back to where I was" (NO5). The desire to get back to work and for normality was characteristic of responses provided by many others. While participants did not articulate concerns about their employer in their ongoing plan, a second subtheme 
TABLE 5: Participants reported symptoms of depression, anxiety and stress as measured by the DASS21 at each follow-up.

\begin{tabular}{lcc}
\hline \multirow{2}{*}{ Symptoms } & \multicolumn{2}{c}{2 weeks $(n=161)$} \\
& $N$ & $\%$ \\
\hline Depression & 114 & 60.8 \\
$\quad$ Normal & 15 & 22.9 \\
Mild & 20 & 8.6 \\
Moderate & 7 & 5.5 \\
Severe & 5 & 1.8 \\
Extremely severe & & \\
Anxiety & 99 & 61.4 \\
Normal & 22 & 13.6 \\
Mild & 21 & 13.0 \\
Moderate & 10 & 6.2 \\
Severe & 9 & 5.6 \\
Extremely severe & & \\
Stress & 98 & 70.8 \\
Normal & 98 \\
Mild & 37 & 9.3 \\
Moderate & 14 & 12.4 \\
Severe & 9 & 4.3 \\
Extremely severe & 3 & 3.1 \\
\hline
\end{tabular}

was also apparent, namely, those whose recovery involved an ongoing plan after a return to health but which emphasised financial and employer support. Such responses included "Having my job held open for me, not working till I am well enough" (RTW12), "Different tasks at work, no heavy lifting" (RTW6), and "Not having the financial burden of not working" (RTW13). Employer support was primarily in relation to workplace accommodations and understanding from their employer, possibly because the nature of their occupation and the injuries sustained would need to be supported by workplace accommodations if a successful return to work was going to be achieved. Injured workers wanted confidence that their employer would do the right thing by them whether it was holding open their job till they were better or providing workplace accommodations like parking or having different duties.

3.8.2. Ideal Recovery Involves a Focus on Return to Health. The second theme covered the majority of open ended responses received by the study participants and strongly focused on factors that would aid their recovery. For this theme, there was no mention of an ongoing plan after a RTH (i.e., plans after recovery). Even though all the participants in this study were in employment, only a proportion of the participants spoke about their employment, financial status, or what support they would need when they get back to work after recovery. Different ways of expressing this theme emerged depending on the importance/focus of factors to the injured participant. These included a return to physical functioning, symptom resolution, mental health, following instructions, and quality of life. Responses were generally positive with an emphasis on themselves and with clear ideas on how they would return to health. Overall planning involved goal setting and intended actions in order to regain a reasonable or preinjury level of physical functioning. While the majority of responses in this theme reflected active engagement by the injured participant in their recovery, a minority $(n=20)$ of the responses seemed unsure of their role in recovery, instead focusing on the actions of others.

Around a quarter of the participants identified complete and partial symptom resolution or a resolution of complications stemming from the injury as important aspects of their return to health plan. "Pins to come out of (leg) and for it (the bone) to knit" (RTW4) "Leg healing, once that is right, I am happy" (NO8). A similar proportion of participants regarding their "return to health plan" mentioned following instructions and/or therapy of their health care providers, "That I follow the therapist's instructions as closely as possible" (NO12). However this participant did not speak of an ongoing plan for after recovery.

For others, the most important thing necessary for their recovery related to healthcare issues. This included access to additional resources "Better sling for my arm, one that doesn't cut the circulation off at my neck" (RTW16) and for others it was communication with their healthcare providers.

Few participants $(<5 \%)$ had a RTH plan that involved consultation with their healthcare providers "Keep going back to Dr for tests and X-rays" (RTW11). Some seemed unsure of their role in their own recovery.

Some injured workers, while making positive statements, had no clear RTH plan. These participants all reported symptoms of depression, anxiety, or stress as measured by the DASS21 two weeks following their injury. When talking about recovery, they were strongly focused on their injury, they did not mention physiotherapy or following the therapists' instructions but appeared more focused on their own mental health such as "being positive during their recovery period" with no mention of plans for "after they have recovered".

3.8.3. Recovery Involves a Focus on Current Status. While many participants indicated the need for rest and time, a minority (12\%) provided comments that suggested they may be "stuck in the moment of the injury and its impact." Regret and generally pessimistic statements served as reminders about the traumatic nature of their injury had restrained their lives "My thumb needs to be perfect for me to do my job properly and I can't see that happening" (NO1), "I need to be allowed to be angry about what has happened" (NO8). For this group of participants, it seemed they were still struggling with the notion that "life would not be the same." Their comments reflected a sense of being "unable to move on" and they appeared in need of psychological assistance. What was noticeable in this theme was the high percentage (90\%) of responses provided by workers who did not RTW during the six month follow-up period. These responses seem similar to the "fall out" phase mentioned in other study described as "an ever increasing awareness of the effect of the injury on their lives." 


\section{Discussion}

Patients' views on recovery following non-life-threatening acute traumatic injuries have received little research attention to date. The aim of the current analysis was to explore patients' views on what would aid their recovery, establish whether there were commonalities in the responses, and determine any change in responses with time following the injury. Participants were asked for a brief response to the following question: "What are the most important things necessary for you to best recover" and what they thought at the time. Responses were received from 168 employed persons who had sustained a range of non-life-threatening acute orthopaedic injuries leading to hospitalization. Analysis of responses revealed common themes regarding participant's perceptions of what they considered "key" to their recovery. The themes derived from the data provide insight into the ways injured people negotiate the recovery process and include having a clear plan for returning to health, a plan for returning to employment, and maintaining recovery strategies including social interaction. Overall the study findings suggest that the most important aspect of recovery for the injured workers was a return to what they perceived as their life before the accident. The attitude of the participant regarding their own recovery seems to influence their immediate decisions regarding return to health and return to work. In particular, returning to "prior to accident" physical functioning, advice from health care specialists, environment provided by the employer, financial situation, and social interactions dominated their responses.

Clay et al. examined the biopsychosocial predictors of RTW using the same dataset as the current study [3]. In that study, participants with strong recovery expectations had 16.7 greater odds of returning to work by 12 weeks than participants with weak recovery expectations. In contrast a Swedish study that examined the association between selfperceived recovery and the SF36 following minor traffic related musculoskeletal injuries found that physical aspects of functional health status were more strongly associated with recovery than emotional or social aspects [26].

The biomedical model of health considers recovery as universal in which all injured persons have a predictable recovery trajectory and "patients" need to follow the instructions of their health care providers to achieve optimal results, sometimes referred to as "structural functionalism" [27]. However our findings and other research studies indicate that while this may be true for a percentage of injured people, recovery overall following injury can be both complex and subjective and may not always follow a predictable trajectory. We would like to emphasise that the use of quotations marks around the responses should not be inferred as representing the injured workers unchanging world view on recovery [28] because as we see in this study, recovery is both individually experienced and a dynamic state.

The recovery process as a continuum influenced the identification of emerging themes that reflected the core thought process of the participant and the different views and perspectives about recovery expectations. For example, we grouped the responses, time and rest because that's how most people think of rehabilitation. Recovery takes time and often it's by resting from the context in which the injury occurred (i.e., work/driving/sport). Had other questions been asked-a limitation of the current study - it is feasible that the grouping of responses may have slightly differed.

This study, while small in nature and exploratory provides evidence to support a relationship between participant responses and time after injury. For example, at the first postinjury time-point (around 2 weeks postinjury), time and rest was the most common response. At this stage many participants remained in the acute/subacute postinjury phase with more than $25 \%$ still in hospital. However, as time passed, a decrease in the frequency of this response was observed. This is perhaps not unexpected in the early postinjury stages given the injury required hospitalisation. That, "time and rest" was offered as a response at later time-points may suggest barriers to ideal recovery for some participants such as depression or chronic pain.

Our findings are similar to another study on the psychological aspects of people with hand injuries under treatment and rehabilitation, which asked its participants the question "What is the single most important thing that will enable the best recovery for you? [29]." Responses in that study included time, caution, attitude to the follow the instructions of the doctor or therapist, and to do the prescribed therapy or exercises for the hand. The study found that caution was considered more important than time in the initial stages. In the current analysis, time and rest was a common and important response. In agreement with our findings, the study by Haese found that responses changed according to the phase of treatment [29]. This is not unusual as recovery is a dynamic process.

Hush et al. (2009) explored patient's perceptions of recovery from low back pain, finding that symptom resolution, and an improved capacity to perform a broad scope of self-defined functional activities were considered important aspects of recovery by the participants [11]. This is in keeping with our findings of the importance of getting back to health and activities following a return to health. In previous studies, psychological health has been identified by participants as a marker of recovery, with particular mention of "having a more positive outlook" and the "importance of not getting depressed". Our study found similar attitudes among participants, for example, "Good healthy outlook, I need to believe I am going to recover." In both studies, psychological health was identified by participants as a marker of recovery, particularly in terms of having a more positive outlook and the importance of not getting depressed.

The focus of the current study was on Victorian workers who were all in salaried employment at the time of their injury. Nearly half were entitled to injury compensation which may have included wage replacement. This may be one reason why the majority of participants were focused on "returning to health" and did not have a "return to work" plan if financial needs could be met via wage replacement. Our study did not specifically ask whether their injury affected their employment; which is a limitation of the study. Work is a central aspect of adult life and provides a sense of social identity [30]. For most, working is a financial necessity 
so recovery [3] and RTW from an unexpected injury are important to workers and their families if only for financial reasons. Murgatroyd et al. (2010) considered the effect of compensation on recovery following severe motor vehicle crash injuries and suggested that recovery was particularly stressful for those claiming compensation [9]. In the current study, around half of the participants were in receipt of injury compensation. Compensable injury status does not appear to have had an impact on participants' views on recovery. While one participant (data not shown) did provide the response of "income support, home support, work support, financial support”, few participants responded similarly and no participant made any direct reference to any compensation system.

The current analysis utilized responses from participants to the question-What are the most important things necessary for you to best recover?. The advantage of opened ended responses is that it permits one to understand the world as seen by the respondents. This enables the researcher to capture the points of view of other people without pre-determining those points of view." [31]. As not all participants provided a response to the open ended question on recovery at each follow-up time-point (see Table 2), the study findings reflect a baseline of concerns. While this needs to be acknowledged as a potential limitation of the study, this baseline of concerns could form the basis of outcome measurements or be explored more rigorously in further studies using in depth interviews that explore in more depth the intricacies associated with recovery. The consistency of some responses for example on workplace and employer accommodations suggests that these issues need further exploration. While government policies and organisations like the Transport Accident Commission encourage communication between employers and employees following injury, our thematic analysis suggests that depending upon the person and the injury, some participants needed more assistance from employers for a successful recovery and RTW than others. This may be due to the nature of their injury or alternately it could be that some people need more assistance than others during the recovery process. This is highlighted by responses such as "Confidence of my employer to look after me" or "Flexibility in working hours, understanding from my employer re mobility restrictions" which reflects their concerns about the circumstances at work. It was not possible to explore these psychological aspects in order to make any inferences with regard to this factor.

The inclusion of pain intensity [6] and participantreported symptoms of depression, anxiety and stress as measured at 2 weeks post-injury reflect the contributions of these factors measured in the early post injury stages as strong predictors of long term recovery $[2,4,5]$. We found participants who reported symptoms of depression, anxiety or stress tended to focus more on their recovery and in their responses did not appear to think about RTW or what they would do after their recovery. This group of participants did not mention what they would do after they recover they seemed to have "no going plan" but were concentrating on their injury status. In addition, our study found that even participants who did not report any symptoms of depression, anxiety or stress as measured by the DASS21 may need some encouragement or guidance to think about an ongoing plan (i.e., plan following recovery or a RTW plan), for example, "Up to the doctors and physios-I don't want to start doing things without seeing the doctor". Bodenheimer et al. (2002) has argued that people with health-related conditions who self-manage their health have an improved chance for a rewarding lifestyle because they take part in their own care [32]. Health behaviour is a product of an individual's feelings, beliefs, and cultural practices. For the injured workers in the current study, moving on and planning were not straightforward. While some within weeks of the injury offered comments on recovery that indicated they were planning beyond the injury, others were focused on a return to health, and a minority overall appeared unable to move from the moment of the injury and its impact.

Research suggests that a single recovery question may be a useful for conducting an overview or overall assessments of recovery following injury [33]. A better understanding of injured workers views on recovery is critical to how recovery is measured in clinical and rehabilitative practice the findings of this study further contribute towards the current debate on how recovery following acute nonlife-threatening traumatic injuries is influenced by more than just physical factors and how recovery could be better measured if the spectrum of factors patients consider key for their recovery are addressed.

4.1. Strengths and Limitations. Statistics derived from quantitative health surveys offer little insight into the human motivation behind certain preferences and behaviours which led us to conclude that a combination of quantitative and qualitative research, would offer a greater breadth of perspectives on the impact of how patients view recovery than just a survey. Participant's perspective on their recovery is one of the strengths of this study as is the use of different methods approach for data collection. Such an approach has provided for greater rigour by reducing the likelihood of "gaps" in the information collected. Other key strengths include its prospective longitudinal design and high followup rate and the measurement of recovery expectations at three post-injury time-points.

Limitations that need to be considered include the need for multiple stages of data collection and the potential loss of flexibility and depth due to quantizing the open text comments [33]. We acknowledge the potential for collinearity in the quantized data. Selection bias may have affected the frequency and type of responses provided at the six month time-point. There was a significant amount of missing data (35\%) at this time point due to participants who felt they had recovered declining to provide a response. We cannot rule out the influence of the healthcare provider on the participant's responses. If a healthcare provider strongly reinforced the message of having to do the therapy or exercise, it is feasible that the participant's responses may not be an unbiased reflection of their own views. Finally, the subjective nature of the recovery comments raises concern of recall bias. While this cannot be ruled out, research has established a high degree of accuracy in recall for important single events including pregnancy and later recall about delivery [34]. We 
assume that injury that results in hospitalization would be an important event and therefore less subject to recall bias.

\section{Conclusions}

In conclusion, the findings of this study indicate that the journey to recovery following non-life-threatening acute orthopaedic trauma is complex, often prolonged, and highly individual. The path can vary considerably between persons who have sustained similar injuries and are influenced by individual perceptions of their own recovery and by family and work situations. These findings need to be confirmed with more homogenous injury populations. They highlight the importance of exploring the patient's views on recovery and provide additional evidence that postinjury recovery cannot easily be explained within a biomedical model that focuses only on factors related to the physical injury. While acknowledging potential methodological limitations, this study has identified some key aspects that injured persons consider will assist their recovery. Some of these aspects are central to the injured person and others to family, treating providers, and the workplace. Further exploration of these aspects and how they affect clinical, rehabilitative, and social outcomes are warranted.

\section{Abbreviations}

RTW: Return to work

RTH: Return to health

NO: No return to work

ISS: Injury Severity Score

VAED: Victorian Admitted Episodes Dataset

VWA: Victorian WorkCover Authority.

\section{Conflict of Interests}

The authors declare that there is no conflict of interests regarding the publication of this paper.

\section{Acknowledgments}

The Transport Accident Commission and the Monash University Accident Research Centre Postgraduate Students Fund funded the data collection phase of this study. The funding bodies played no role in the design, conduct, analysis or interpretation of the data or in the writing of this paper. Fiona J. Clay was the recipient of an NHMRC Public Health scholarship and VIC-Health scholarship and a participant in the CIHR strategic training program in Work Disability Prevention Program at the time of data collection.

\section{References}

[1] D. M. Urquhart, O. D. Williamson, B. J. Gabbe et al., "Outcomes of patients with orthopaedic trauma admitted to level 1 trauma centres," ANZ Journal of Surgery, vol. 76, no. 7, pp. 600-606, 2006.

[2] D. Zatzick, G. J. Jurkovich, F. P. Rivara et al., "A national US study of posttraumatic stress disorder, depression, and work and functional outcomes after hospitalization for traumatic injury," Annals of Surgery, vol. 248, no. 3, pp. 429-435, 2008.

[3] F. J. Clay, S. V. Newstead, W. L. Watson, J. Ozanne-Smith, J. Guy, and R. J. McClure, "Bio-psychosocial determinants of persistent pain 6 months after non-life-threatening acute orthopaedic trauma," Journal of Pain, vol. 11, no. 5, pp. 420-430, 2010.

[4] E. J. MacKenzie, M. J. Bosse, J. F. Kellam et al., "Early predictors of long-term work disability after major limb trauma," Journal of Trauma, vol. 61, no. 3, pp. 688-694, 2006.

[5] M. Fitzharris, D. Bowman, and K. Ludlow, "Factors associated with return-to-work and health outcomes among survivors of road crashes in Victoria," Australian \& New Zealand Journal of Public Health, vol. 34, no. 2, pp. 153-160, 2010.

[6] F. J. Clay, S. V. Newstead, W. L. Watson, and R. J. McClure, "Determinants of return to work following non-life-threatening acute orthopaedic trauma: a prospective cohort study," Journal of Rehabilitation Medicine, vol. 42, no. 2, pp. 162-169, 2010.

[7] M. V. Mondloch, D. C. Cole, and J. W. Frank, "Does how you do depend on how you think you'll do? A systematic review of the evidence for a relation between patients' recovery expectations and health outcomes," Canadian Medical Association Journal, vol. 165, no. 2, pp. 174-179, 2001.

[8] D. Ozegovic, L. J. Carroll, and J. David Cassidy, “Does expecting mean achieving? the association between expecting to return to work and recovery in whiplash associated disorders: a population-based prospective cohort study," European Spine Journal, vol. 18, no. 6, pp. 893-899, 2009.

[9] D. F. Murgatroyd, I. D. Cameron, and I. A. Harris, "Understanding the effect of compensation on recovery from severe motor vehicle crash injuries: a qualitative study," Injury Prevention, vol. 17, no. 4, pp. 222-227, 2011.

[10] T. S. Richmond, H. J. Thompson, J. A. Deatrick, and D. R. Kauder, "Journey towards recovery following physical trauma," Journal of Advanced Nursing, vol. 32, no. 6, pp. 1341-1347, 2000.

[11] J. M. Hush, K. Refshauge, G. Sullivan, L. De Souza, C. G. Maher, and J. H. McAuley, "Recovery: what does this mean to patients with low back pain?" Arthritis Care and Research, vol. 61, no. 1, pp. 124-131, 2009.

[12] D. E. Beaton, V. Tarasuk, J. N. Katz, J. G. Wright, and C. Bombardier, "“'Are you better?" A qualitative study of the meaning of recovery", Arthritis Care and Research, vol. 45, no. 3, pp. 270-279, 2001.

[13] G. L. Engel, "The need for a new medical model: a challenge for biomedicine," Science, vol. 196, no. 4286, pp. 129-136, 1977.

[14] Department Human Services, The Victorian Admitted Episode Database: An Overview, Acute Health Division, Department of Human Services, Melbourne, Australia, 14th edition, 2004.

[15] National Centre for Classification in Health, The International Statistical Classification of Disease and Related Health ProblemsAustralian Modification (ICD-10-AM), Tenth Revision Sydney: National Centre for Classification in Health, 6th edition.

[16] Association for the Advancement of Automotive Medicine, The Abbreviated Injury Scale 1990, Barrington, Ill, USA, 1998.

[17] S. P. Baker, B. O’Neill, W. Haddon Jr., and W. B. Long, "The injury severity score: a method for describing patients with multiple injuries and evaluating emergency care," Journal of Trauma, vol. 14, no. 3, pp. 187-196, 1974.

[18] R. Melzack, "The short-form McGill pain questionnaire," Pain, vol. 30, no. 2, pp. 191-197, 1987.

[19] M. P. Jensen, P. Karoly, and S. Braver, "The measurement of clinical pain intensity: a comparison of six methods," Pain, vol. 27, no. 1, pp. 117-126, 1986. 
[20] P. F. Lovibond and S. H. Lovibond, “The structure of negative emotional states: comparison of the depression anxiety stress scales (DASS) with the Beck Depression and Anxiety Inventories," Behaviour Research and Therapy, vol. 33, no. 3, pp. 335-343, 1995.

[21] S. Lovibond and P. Lovibond, Manual for the Depression Anxiety Stress Scale, The Psychology Foundation Monograph, Sydney, Australia, 2nd edition, 1995.

[22] D. C. Cole, M. V. Mondloch, and S. Hogg-Johnson, "Listening to injured workers: how recovery expectations predict outcomesa prospective study," CMAJ, vol. 166, no. 6, pp. 749-754, 2002.

[23] A. G. Tuckett, "Applying thematic analysis theory to practice: a researcher's experience," Contemporary Nurse, vol. 19, no. 1-2, pp. 75-87, 2005.

[24] J. W. Cresswell, Qualitative Inquiry and Research Design: Choosing among Five Approaches, Sage, Thousand Oaks, Calif, USA, 2nd ed edition, 2007.

[25] World Medical Organization, "Declaration of Helsinki," British Medical Journal, vol. 313, no. 7070, pp. 1448-1449, 1996.

[26] C. Ottosson, H. Pettersson, S.-E. Johansson, O. Nyrén, and S. Ponzer, "Recovered? Association between self-perceived recovery and the SF-36 after minor musculoskeletal injuries," Quality of Life Research, vol. 16, no. 2, pp. 217-226, 2007.

[27] T. Parsons, "Social structure and dynamic process: the case of modern medical practice," in The Social System, chapter 10, Routledge, London, USA.

[28] S. Taylor, "One participant said ...: the implications of quotations from biographical talk," Qualitative Research, vol. 12, pp. 388401, 2012.

[29] J. B. Haese, "Psychological aspects of hand injuries their treatment and rehabilitation," Journal of Hand Surgery, vol. 10, no. 3, pp. 283-287, 1985.

[30] M. Corkum, "Mental health: together we do better: marketing meets mental health promotion and work," in Australian Network for Promotion Prevention and Early Intervention for Mental Health. Mental Health and Work: Issues and Perspectives, E. Willis, L. Morrow, and I. Verins, Eds., pp. 165-178, Auseinet, Bedford Park, UK, 2002.

[31] M. Q. Patton, Qualitative Evaluation and Research Methods, Sage, Newbury Park, Calif, USA, 2nd edition, 1990.

[32] T. Bodenheimer, K. Lorig, H. Holman, and K. Grumbach, "Patient self-management of chronic disease in primary care," Journal of the American Medical Association, vol. 288, no. 19, pp. 2469-2475, 2002.

[33] C. W. Roberts, "A conceptual framework for quantitative text analysis: on joining probabilities and substantive inferences about texts," Quality and Quantity, vol. 34, no. 3, pp. 259-274, 2000.

[34] M. A. Quigley, C. Hockley, and L. L. Davidson, "Agreement between hospital records and maternal recall of mode of delivery: evidence from 12391 deliveries in the UK Millennium cohort study," BJOG, vol. 114, no. 2, pp. 195-200, 2007. 


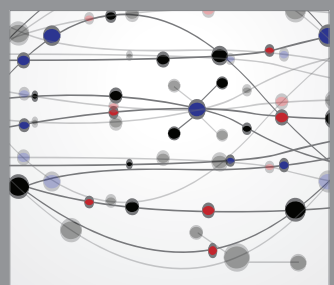

The Scientific World Journal
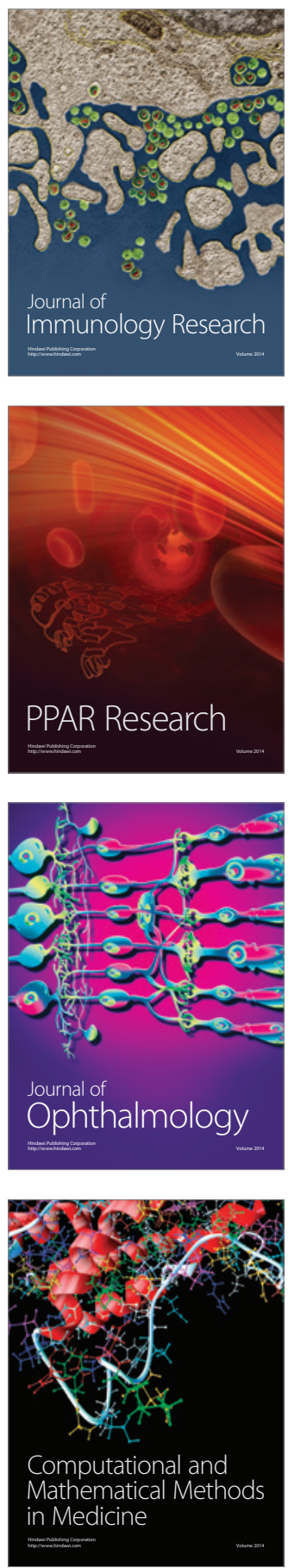

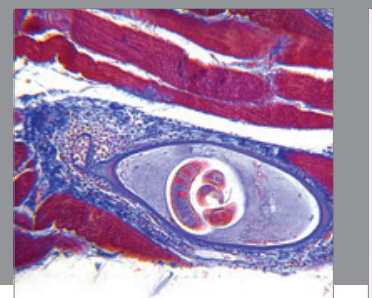

Gastroenterology

Research and Practice
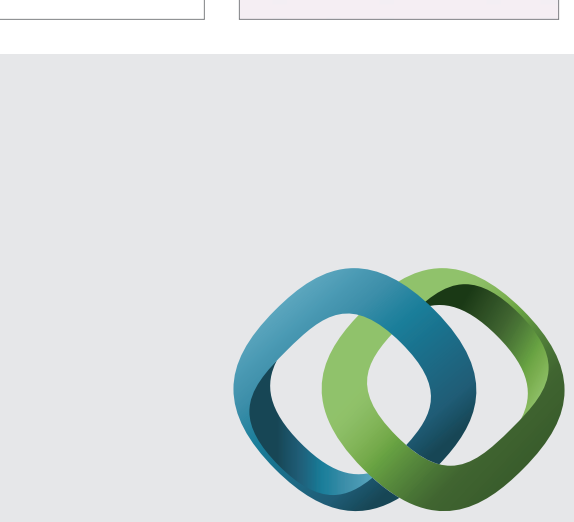

\section{Hindawi}

Submit your manuscripts at

http://www.hindawi.com
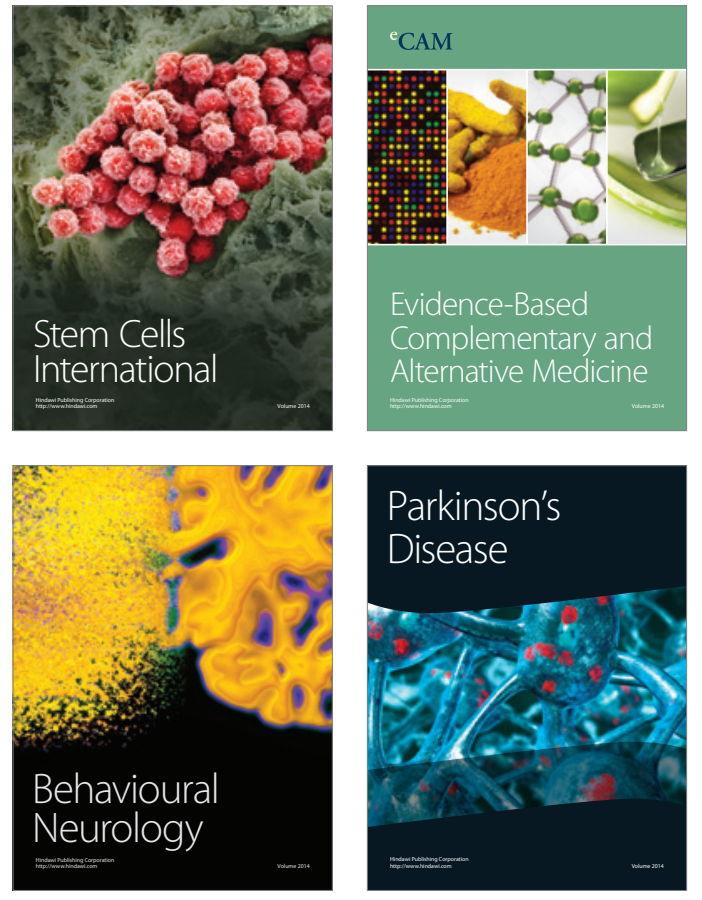
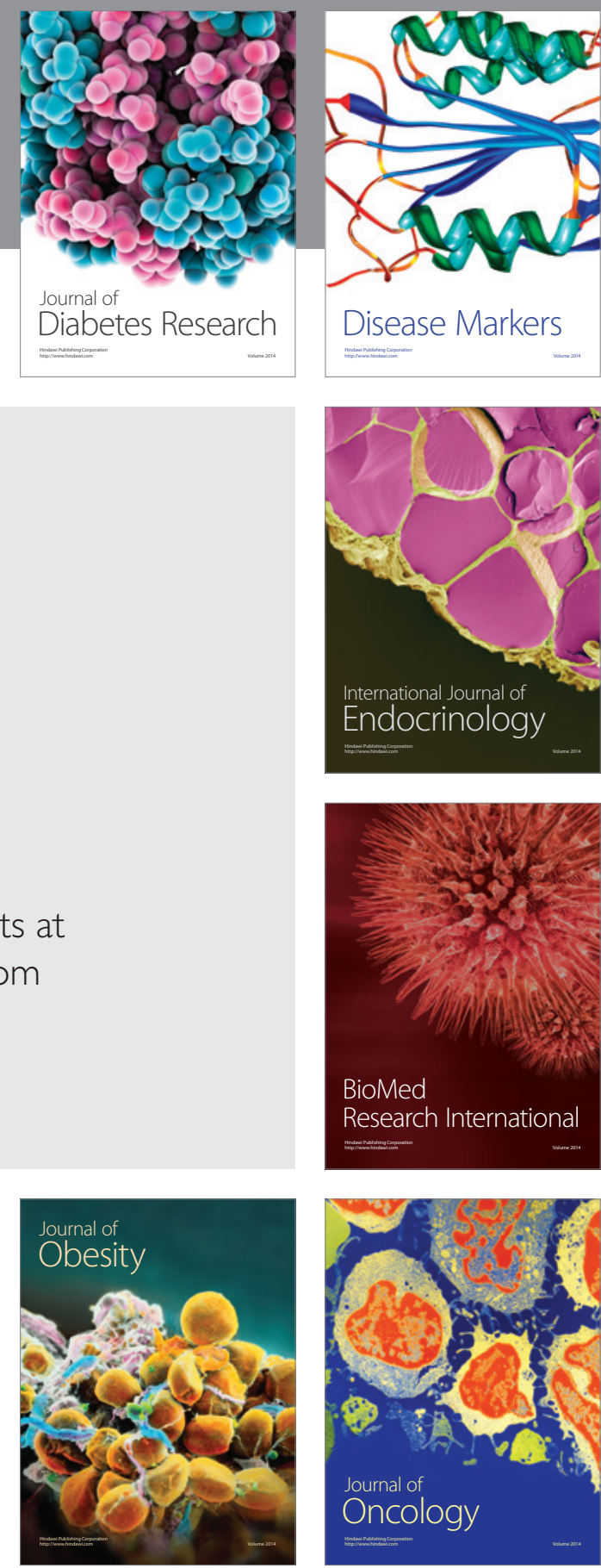

Disease Markers
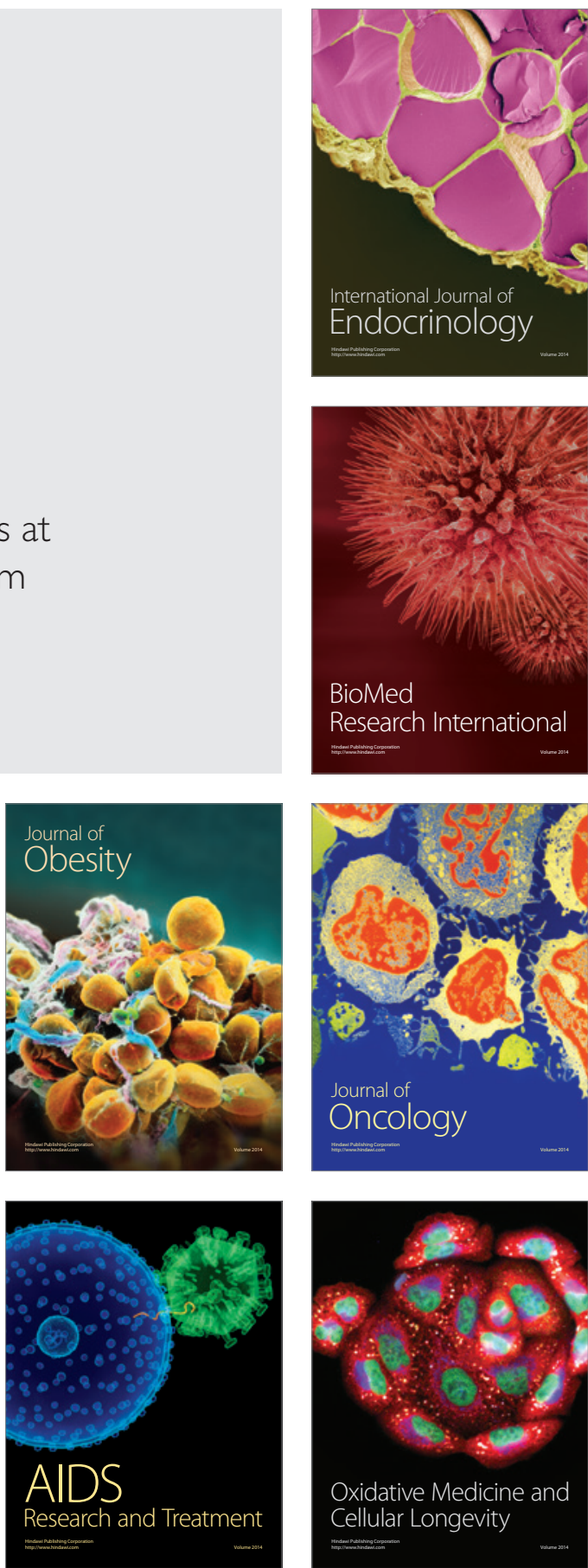